\title{
c-fos Expression in Vomeronasal Pathways of Mated or Pheromone-stimulated Male Golden Hamsters: Contributions from Vomeronasal Sensory Input and Expression Related to Mating Performance
}

\author{
Gwendolyn D. Fernandez-Fewell and Michael Meredith \\ Program in Neuroscience, Department of Biological Science, Florida State University, Tallahassee, Florida 32306
}

The vomeronasal system projects to the accessory olfactory bulb (AOB), to the medial (Me) and posterior medial cortical nuclei (PMCN) of the amygdala, to the bed nucleus of the stria terminalis (BNST), and to other central structures shown to be important in mating behavior, including the medial preoptic area (MPOA). In these experiments c-fos expression was used as a marker of neural activity to identify the contribution of vomeronasal sensory input during mating behavior in male golden hamsters, either intact or with vomeronasal organs removed (VNX). Inexperienced hamsters were either stimulated with a receptive female and allowed to mate, exposed to female hamster vaginal fluid (HVF), which contains stimuli known to act through the VN system, or placed in a clean cage alone. Densely stained Fos-positive nuclei were evident in mated animals in the central VN pathway [AOB, Me, posterior medial BNST (pmBNST)] and a VN target area (MPOA). HVF-exposed animals showed Fos expression in the AOB, Me, and BNST but not MPOA. Unstimulated animals showed almost no activation. Most VNX animals exposed to females did not mate, but performed intense chemoinvestigation. They had few Fos-positive nuclei in any of these areas except the caudal pmBNST. A few VNX animals that did mate had patterns of Fos activation that were similar but less intense than those of intact mating animals, suggesting a selective activation of VN central pathways during mating regardless of VN sensory input. The main olfactory system showed low levels of Fos expression in all animals (stimulated and unstimulated). Fos expression in the MPOA and rostral pmBNST was seen only in mated animals, suggesting that these regions are concerned with mating performance or its consequences, rather than the chemosensory input that triggers it. Fos expression in the caudal encapsulated pmBNST was evident in all groups of animals that performed chemosensory investigation, regardless of VN status or mating, suggesting that this region either directs or responds to chemosensory investigation.

\footnotetext{
Received July 29, 1993; revised Oct. 20, 1993; accepted Nov. 24, 1993.

We thank Alice G. Howard for assistance with histology, Dr. C. Ouimet for use of the vibratome, Charles Badland for photographic assistance, and Richard Brunck for assistance with illustrations. We also thank an anonymous reviewer for helpful suggestions. This work was supported by NIH Grant DC 00906.

Correspondence should be addressed to Dr. Michael Meredith, Department of Biological Science, Florida State University, Tallahassee, FL 32306-4075.

Copyright (C) 1994 Society for Neuroscience $0270-6474 / 94 / 143643-12 \$ 05.00 / 0$
}

[Key words: vomeronasal, Fos, sex behavior, hamster, accessory olfactory, chemosensory, amygdala, bed nucleus stria terminalis, medial preoptic]

The vomeronasal (VN) or accessory olfactory system is a second chemosensory system organized in parallel with the main olfactory system, and with anatomically distinct pathways (Scalia and Winans, 1975; Davis et al., 1978). The vomeronasal organs (VNOs) are tubular structures lying bilaterally in the ventral part of the nasal cavity. Vomeronasal receptor neurons project to the accessory olfactory bulb (AOB) (Barber and Raisman, 1974) from which fibers project to two parts of the amygdala, the medial nucleus (Me) and the posterior medial cortical nucleus (PMCN), collectively called the vomeronasal amygdala (Kevetter and Winans, 1981a). The vomeronasal amygdala, and the $\mathrm{AOB}$ in some species, projects via the stria terminalis to the medial bed nucleus of the stria terminalis (mBNST) (Scalia and Winans, 1976; Davis and Macrides, 1978; Lehman and Winans, 1983). The Me also has projections via the ventral amygdaloid pathway and the stria terminalis to the medial preoptic anterior hypothalamic area and BNST (Lehman and Winans, 1983), and these regions also project to the ventromedial nucleus of the hypothalamus ( $\mathrm{VmH}$ ). Thus, the accessory olfactory system has relatively direct and restricted projections to central structures known to be involved in reproductive physiology and behavior. In contrast, the second-order projections of the main olfactory system spread widely and make somewhat less direct connections to structures related to reproduction. The main olfactory bulb projects to the anterior olfactory nuclcus and olfactory tubercle as well as to pyriform, entorhinal, and lateral amygdaloid regions (Scalia and Winans, 1976; Davis et al., 1978). Nevertheless, there are projections from the lateral (olfactory) amygdala to the vomeronasal amygdala (Krettek and Price, 1978; Kevetter and Winans, 1981b). Neurons in the vomeronasal amygdala can be driven by separate stimulation of both olfactory and vomeronasal systems, suggesting a possible functional convergence in this region (Licht and Meredith, 1987). The importance of the VN system has been demonstrated by a number of lesion and behavioral studies implicating this system in several aspects of chemical communication and reproductive behavior in several species. In rodents, chemical signals acting through the VNO affect male sexual behavior (Powers and Winans, 197.5; Wysocki et al., 1982; Meredith, 1986) and hormonal responses to the opposite sex (Wysocki et al., 1983; Coquelin 
et al., 1984), as well as some aspects of female sexual behavior (Saito and Moltz, 1986), estrus cycling (Reynolds and Keverne, 1979; Sanchez-Criado, 1982), the timing of puberty (Lomas et al., 1982; Sanchez-Criado, 1982), and in some circumstances, ovulation and pregnancy (Lloyd Thomas et al., 1982; Beltramino et al., 1983). Disruption of VN input to the CNS inhibits some or all of these responses in various species (Meredith, 1983; Wysocki and Meredith, 1987).

We present here results from experiments designed to identify vomeronasal and other contributions to central brain regions activated during mating behavior or exposure to female chemosignals in male hamsters. We used c-fos expresssion (Morgan et al., 1987; Sagar et al., 1988) as a marker of neuronal activation, identifying activated neurons in the brain and olfactory bulbs by Fos protein immunocytochemistry. Fos expression was analyzed following mating behavior, exposure to female hamster vaginal fluid (HVF), and in control conditions in both intact animals and in animals whose vomeronasal organs had been removed (VNX). Hamsters are very dependent on chemosensory input for mating behavior, but other inputs may also affect c-fos expression patterns during mating. Fos activation could be attributable to chemosensory inputs, other sensory inputs, or mating-related inputs including the motor and integrative aspects of behavioral performance. Female HVF contains pheromonal stimuli known to act through the vomeronasal system (Clancy et al., 1984). It has both attractant and aphrodisiac (pheromonal) properties that can elicit mating behavior in male hamsters (Johnston, 1977), but alone it provides no opportunity for mating performance. Animals exposed to HVF have the stimulus largely restricted to the $\mathrm{VN}$ and main olfactory systems. Activity related to mating performance and female cues other than HVF can be eliminated in this way. Analysis of Fos expression patterns in groups of animals with different sensory inputs and/or behaviors helps to visualize contributions from $\mathrm{VN}$ and non-VN sensory inputs and from nonsensory activation. Activity due to nonvomeronasal sensory input was analyzed in VNX animals stimulated with a female or HVF. Although most VNX animals do not mate, some do and their $\mathrm{c}$-fos expression pattern can be attributed to mating-related activation and non-VN inputs. Unstimulated animals were placed in clean cages without a female or HVF, so any activation in these animals is unlikely to reflect mating or specific chemosensoryrelated events.

Our results in intact animals allowed to mate are similar to those reported in males of other species and show some similarity to results in mating females. However, our use of vomeronasal lesions to explore contributions of VN sensory input in males is unique among reports on Fos expression patterns following male mating behavior. It reveals that despite the selectivity of VN pathway activation in mating and HVF-exposed animals, and despite the evidence for a VN contribution to that activation, VN sensory input is not essential for much of the activation. Our data suggest that Fos expression in the Me has both vomeronasal and nonvomeronasal components, including a strong contribution from mating performance. Fos expression in the MPOA (medial preoptic arca) and the rostral part of the posteromedial BNST (pmBNST) depends critically on the involvement of mating performance and appears to have little contribution from VN sensory input. On the other hand, Fos expression in the caudal part of the pmBNST appears to be related to chemoinvestigatory behavior regardless of the achievement of mating performance.

\section{Materials and Methods}

Sexually inexperienced Spraguc-Dawley male golden hamsters (Mesocricetus auratus), bred in the laboratory, were maintained on a reversed $14 \mathrm{~L} / 10 \mathrm{D}$ light cycle. Six groups of animals were used to study the pattern of activation of various brain regions by vomeronasal input. These were intact mated (Ism, $n=8$ ), intact HVF exposed (Is HVF, $n$ $=4$ ), intact unstimulated (Ius, $n=7$ ), and equivalent groups with vomeronasal organs removed (VNX); VNX stimulated (Vs, $n=7$ ), bilateral VNX, HVF stimulated (Vs HVF, $n=3$ ), unilateral VNX, HVF stimulated (Vs uni, $n=3$ ), and VNX unstimulated (Vus, $n=10$ ). Most VNX animals did not mate, but two additional VNX animals did mate and are presented as a separate group: VNX animals that mated (Vsm). All VNX animals had their vomeronasal organs (VNOs) removed at $17 \mathrm{~d}$ when it was easier to remove all of the organ. The procedure has previously been shown to give reliable deficits similar to those resulting from adult removal (Meredith, 1986; Fernandez and Meredith, 1991). Littermates were assigned randomly to VNX and intact surgical groups. VNOs were excised through the palate under sterile conditions while the animals were under pentobarbital anesthesia $(80 \mathrm{mg} / \mathrm{kg}$, i.p.). The palate was then closed with cyanoacrylate tissue adhesive and left to heal. All experimental animals were tested behaviorally at 2-3 months of age. For behavioral testing, the sexually inexperienced male hamsters, both intact and VNX, were placed in a clean plastic box $42 \mathrm{~cm} \times 20$ $\mathrm{cm} \times 20 \mathrm{~cm}$ with fresh bedding and left for 1 min to get accustomed to their surroundings. They were then presented successively with two or three naturally cycling, behaviorally receptive females and allowed to mate for $45 \mathrm{~min}$. The female was changed once or twice for maximal stimulation. After $45 \mathrm{~min}$ of mating, the male was left alone for another $45 \mathrm{~min}$ before being killed with an overdose of sodium pentobarbital and perfused for c-fos immunocytochemistry. For stimulation with HVF a similar procedure was used, except that male hamsters were presented with HVF, approximately $0.2 \mathrm{ml}$ diluted $1: 10$ with distilled water placed in a glass well slide and replenished four or five times, for up to $45 \mathrm{~min}$, after which the animal was left alone for $45 \mathrm{~min}$ before being killed and perfused for c-fos immunocytochemistry. Controls, that is, unstimulated, intact, and VNX animals, were placed in a clean box with fresh bedding for 90 min and, as for the stimulated animals, the cage lid was opened at least twice providing some mildly arousing stimulus during this period. Animals for assignment to treatment groups were picked at random except for the constraint that we end up with approximately equal numbers of animals in the experimental groups.

\section{Immunocytochemical procedures}

The deeply anesthetized animals were perfused through the heart with $0.1 \mathrm{M}$ phosphate-buffered saline ( $\mathrm{pH} 7.4$ ), followed by fixation with $4 \%$ paraformaldehyde (pH 7.3-7.4). The brain along with olfactory bulbs was removed. The olfactory bulbs were separated and embedded in gelatin ( 300 bloom) and both the brain and bulbs were postfixed overnight in the same fixative solution. The noses of VNX animals were postfixed separately and later decalcified and sectioned for verification of the lesion. The brain and olfactory bulbs were cut serially on the vibratome at $40 \mu \mathrm{m}$ thickness ( $50 \mu \mathrm{m}$ for a few animals). Free-floating coronal sections of the brain and horizontal sections of the bulbs were washed in $0.1 \mathrm{M}$ phosphate buffer and incubated with Fos polyclonal antibody made in sheep (Cambridge Research Biochemicals OA-11823 ), at a dilution of $1: 40,000$ in phosphate-buffered saline (PBS) with $0.4 \%$ Triton $\mathrm{X}-100$, for $48 \mathrm{hr}$ at $4^{\circ} \mathrm{C}$. This affinity-purified polyclonal antibody has been shown to detect Fos and Fos-related proteins; therefore, in our results, the term "Fos" can be considered to include Fosrelated proteins. After rinsing in $0.1 \mathrm{M}$ phosphate buffer the sections were incubated in biotinylated secondary antibody, rabbit anti-sheep IgG (heavy and light chains; Vector Labs) diluted 1:600 in PBS with $0.4 \%$ Triton X-100, for $1 \mathrm{hr}$ at room temperature; rinsed in $0.1 \mathrm{M}$ phosphate buffer; and then incubated in an avidin-biotin HRP conjugate ( $A B C$ standard-elite kit, Vector Labs), at a dilution of 1 drop solution A +1 drop solution B per $10 \mathrm{ml}$ of $10 \mathrm{mM}$ PBS, for $1 \mathrm{hr}$ at room temperature. Sections were washed in $0.1 \mathrm{~m}$ phosphate buffer, and the Fos antibody-peroxidase complex was stained with 3,3'-diaminobenzidine (DAB) for $5 \mathrm{~min}$. The stain was either brown, without nickel intensification, or black, with nickel chloride intensification (DAB kit, Vector Labs). A few animals had alternate sections stained black and brown. Following staining with $\mathrm{DAB}$, the sections were washed in 0.1 M phosphate buffer (three changes) and then mounted on gelatin/chrome alum-coated glass slides, air dried, dehydrated through graded ethanol 
solutions, cleared in xylene, and coverslipped with Permount. The presence of Fos was evident as a brown or black (nickel intensified) reaction product in cell nuclei.

Controls for specificity included negative (without primary antibody) and preabsorption controls, which involved incubation of sections in antisera that had been preabsorbed with $10 \mathrm{mg}$ of Fos antigen per milliliter of diluted antiserum (Cambridge Research Biochemicals; Beltz and Burd, 1989). These controls did not show any staining.

Fos expression in experimental animals was examined in every section or in every other section throughout much of the forebrain. The following regions of interest showed reliable differences in Fos expression between our experimental groups and were studied extensively in each animal: accessory olfactory bulb, medial amygdala, medial preoptic area/anterior hypothalamus, bed nucleus of the stria terminalis (both anterior medial and posterior medial), and paraventricular thalamus. Representative sections showing the differences between groups are illustrated in the figures. These differences were quantified by counting all Fos-expressing nuclei within one equivalent single section for each region of interest in each experimental animal from all groups. Equivalent sections were selected on the basis of neuroanatomical landmarks. The number of nuclei in each neuroanatomical nucleus was counted visually at $160 \times$ magnification and the numbers then averaged across all the animals in that group. The illustrations of Kollack and Newman (1992) and a series of cresyl violet-stained serial sections through the male hamster brain were used as guidelines for nuclear boundaries. The cross-sectional areas of the different brain regions where nuclei were counted vary between regions but are the same for a given region in all animals. Comparisons between patterns of activity across the same brain regions in different animal groups are instructive. Where our animal treatments are similar to those of previously published reports, both the pattern and the relative increase in number of Fos-positive nuclei with treatment appear to be similar (Kollack and Newman, 1992; Baum and Wersinger, 1993; Fiber et al., 1993), although the absolute number of Fos-positive nuclei in both treated and control animals was less than in some reports.

Differences in counts of Fos-positive nuclei between the six groups of animals were analyzed using a fully factorial analysis of variance (ANOVA), for each area of the brain, as a function of treatment (female stimulated, HVF stimulated, unstimulated) and surgery (intact, VNX), followed by post hoc Tukey tests. All comparisons noted as significant had probabilities lower than $p=0.01$. Additional tests between counts for the VNX animals that mated (Vsm) and other groups, as well as differences between intact and VNX sides for unilaterai VNX animals, used the Mann-Whitney test, which can provide probability levels ( $p$ $<0.05$ here) for small group data (Siegel, 1956).

Histology. Noses of VNX animals were decalcified in RDO (Apex Engineering, Plainfield, IL) for $8 \mathrm{hr}$, dehydrated, embedded in paraffin, and cut at $15 \mu \mathrm{m}$ coronally. Serial sections through the region of the vomeronasal organ were examined for signs of residual VN tissue and the rest of the nose examined for signs of damage to the main olfactory system or other structures.

Line drawings. Microprojector images of representative brain sections contributing to the data were traced and then scanned into a computer, to make the line drawings of whole brain sections. These drawings are used to illustrate the position of the photomicrographs in different brain regions.

\section{Results}

\section{Fos expression during mating behavior}

In intact mated male hamsters, intense c-fos expression appeared selectively in the central accessory olfactory (vomeronasal) pathway after mating behavior (Fernandez and Meredith, 1992). Intense Fos protein immunoreactivity was seen in the accessory olfactory bulb, medial amygdala, bed nucleus of the stria terminalis, and medial preoptic area of intact stimulated (Ism) animals, all of which mated with the female for much of the 45 min exposure time. Much less Fos immunoreactivity was seen in intact unstimulated (Ius) animals and VNX stimulated (Vs) animals, and practically none was seen in VNX unstimulated (Vus) animals (Figs. 1-5). Most VNX animals, seven of nine that were stimulated with females, did not mate, but two of nine did and are described separately. In all VNX animals, the vomeronasal nerve layer and glomerular layer of the accessory olfactory bulb were dramatically reduced and glomeruli were not visible. The patterns of Fos expression in the different groups are described below.

In the $\mathrm{AOB}$, the mitral $(\mathrm{mcl})$ and granule $(\mathrm{gcl})$ cell layers were activated in intact mated animals (Fig. $1 A$ ). Intact unstimulated animals (Fig. $1 B$ ) showed some activation in these areas but the activation is much less than the intact mated group. The VNX stimulated animals show even less activation (Fig. $1 C$ ) and the VNX unstimulated animals showed practically no activation (Fig. 1D; see Fig. 3). VNX stimulated animals that mated did not have many Fos-positive nuclei in either the mitral or granule cell layer in the $\mathrm{AOB}$ and the activation was at the same low level seen in VNX stimulated animals that did not mate, or in unstimulated VNX controls. Fos-stained nuclei were also observed in the main olfactory bulb, mainly in the granule cell layer; however, there were no systematic differences in this Fos staining between the stimulated and unstimulated groups.

The medial amygdala in intact mated animals had a very high level of Fos expression and showed a characteristic pattern of activation. Two distinct clusters of nuclei appeared in the dorsal posterior medial region, which were essentially identical in all intact stimulated males (Fig. $2 A$ ). VNX stimulated animals that did not mate (Fig. $2 C$ ) showed more activation within the medial amygdala than unstimulated animals (Fig. $2 B, D$ ) but distinctly less than intact stimulated animals, as shown by the bar graph (Fig. 3), and they lacked the characteristic clusters seen in intact stimulated animals. VNX stimulated animals that did mate had a higher level of Fos expression in the Me, and one animal also showed the two clusters characteristic of intact mated animals. The number of Fos-positive cells was still significantly lower than in intact mated animals, and significantly higher than in VNX stimulated animals that did not mate.

Intense Fos expression was observed in the medial preoptic/ anterior hypothalamic area (MPOA/AH) in intact mated animals as illustrated in Figure $4 A$. Both the dorsal part bordering on the pmBNST and ventral parts showed intense staining and both are included in the counts (Fig. 3). Dense Fos staining continued rostrally into the medial preoptic nucleus. In contrast, VNX stimulated nonmating animals had few Fos-stained nuclei in the MPOA/AH while unstimulated animals had practically none (Fig. $4 B-D$ ). Note that VNX stimulated animals shown in these figures did not mate although they were exposed to females for $45 \mathrm{~min}$, and did perform chemosensory investigative behaviors. Unstimulated animals were not exposed to females. The two VNX stimulated animals that did mate showed significantly more activation in the MPOA/AH than VNX stimulated animals that did not mate, but again the total count of cells activated was significantly lower than in intact stimulated animals (Fig. 3).

The pattern of activation across groups in the medial bed nucleus of the stria terminalis (mBNST) was largely similar to that in the MPOA/AH, with intense Fos expression observed in intact stimulated animals, less in VNX stimulated animals, and practically no expression in unstimulated animals (Figs. 3, $5 A-D$ ). Intact stimulated animals show some activation in the anterior $\mathrm{mBNST}$, as well as a characteristic pattern of activation with two clusters of nuclei in the rostral part of the posterior mBNST (Fig. $5 A$ ). The VNX stimulated nonmating group lacked the characteristic clusters and showed very little Fos expression rostrally (Fig. $5 C$ ), but surprisingly did show Fos-stained nuclei in the caudal posterior mBNST (Fig. 6D) similar to that in intact 

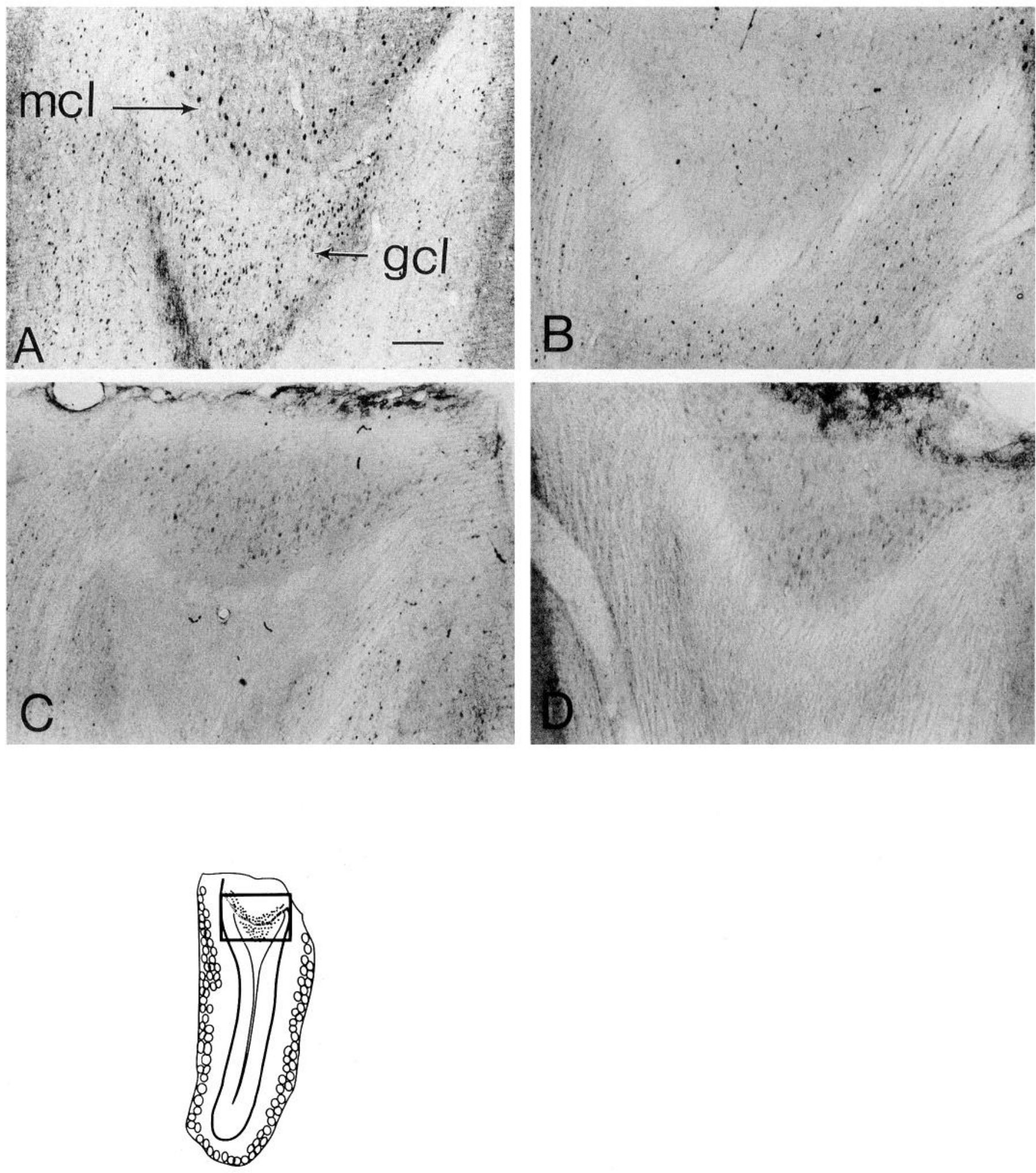

Figure 1. $A-D$, Horizontal sections ( $50 \mu \mathrm{m}$ vibratome) at equivalent levels through the accessory olfactory bulb: intact stimulated $(A)$, intact unstimulated $(B)$, VNX stimulated $(C$, and VNX unstimulated animals $(D)$, stained for Fos protein. Both the mitral and granule cell layers show Fos-positive nuclei in intact stimulated animals $(A)$. VNX stimulated and unstimulated animals do not show much activation in these layers $(C$, $D$ ). Intact unstimulated animals (not exposed to a receptive female; $B$ ) show some activation but less than intact stimulated animals. See bar graphs, Figure 3. Diagrams at the bottom left of this and following figures were made of actual sections from which counts were made and indicate the position of the photomicrographs seen above. $\mathrm{mcl}$, mitral cell layer; $\mathrm{gcl}$, granule cell layer. Scale bar, $100 \mu \mathrm{m}$ for all panels. 

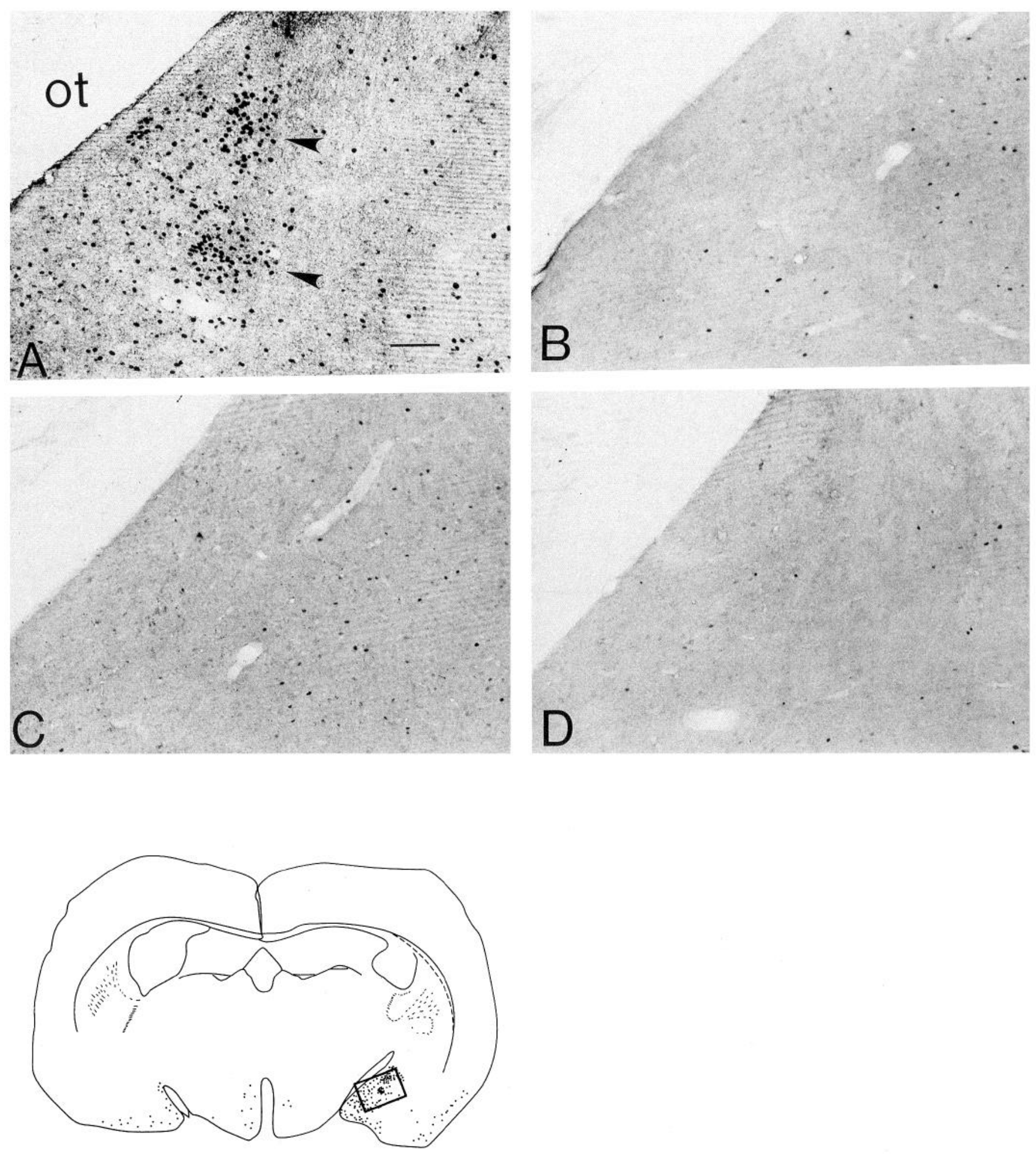

Figure 2. $A-D$, Coronal sections through the medial amygdala, stained for Fos protein at the level of the optic tract. Dorsal is to upper right (see diagram). $A$, Intact stimulated animal; the two clusters of activated cells (arrowheads) are characteristic of intact stimulated animals. $B$, Intact unstimulated animal. $C$, VNX stimulated animals show more activation than unstimulated animals $(B$, intact unstimulated; D, VNX unstimulated), but significantly less than intact stimulated animals $(A)$, and lack the characteristic clusters. ot, optic tract. Scale bar, $100 \mu \mathrm{m}$.

mated animals (Fig. 3). VNX animals that mated (Vsm) had activated nuclei in both the rostral and caudal regions of the posterior medial BNST (pmBNST) not significantly different from that in intact mated animals (Fig. 3). There were, however, significant differences in the rostral pmBNST between mating and nonmating animals and in the caudal pmBNST between stimulated and unstimulated animals regardless of whether they mated. 


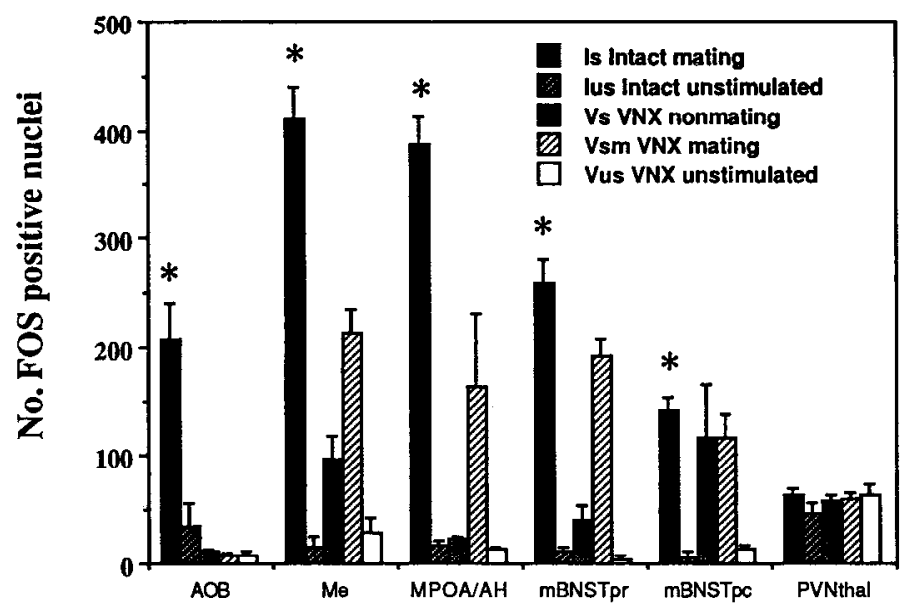

Figure 3. Bar graph showing counts of Fos-positive nuclei comparing intact and VNX stimulated mating and nonmating animals. Intact stimulated $(I s)$ animals show significantly greater $\left(^{*}, p<0.01\right)$ Fos expression within the AOB, Me, MPOA/AH, and BNST (anterior and posterior) compared with VNX stimulated nonmating $(V S)$ and unstimulated animals $(I u s, V u s)$. VNX stimulated animals that mated $(V s m)$ showed significantly more $(p<0.05)$ Fos expression than VNX animals that did not mate in all areas except in the AOB. Vsm animals also had practically no Fos expression in the AOB and significantly less $(p<$ $0.05)$ Fos expression within Me compared to intact mated animals.

Some Fos-expressing cells were also observed in the paraventricular nucleus of the hypothalamus in intact and VNX mating animals, but not in any other group. Other areas expressing low to moderate levels of Fos in intact stimulated animals were the diagonal band of Broca, medial septum, and the anterior olfactory nucleus. VNX nonmating and unstimulated animals showed much less or no Fos expression in these areas.

\section{Fos expression in $H V F$-stimulated animals}

Intact male hamsters exposed to female HVF had a pattern of Fos expression in central VN pathways similar to that described in intact mated animals. However, the overall numbers of activated neurons in the AOB, Me, and BNST were less than those in intact mated animals (Fig. 7A). In HVF-stimulated animals the number of activated cells in the accessory olfactory bulb, while displaying a similar pattern of Fos expression to that in mated males, was smaller in both mitral and granule cell layers (Fig. 6A). In the medial amygdala there were nuclei with intense Fos expression; however, the characteristic clusters observed in intact mated animals in the posterior dorsal Me were absent in HVF-exposed animals (Fig. 6B). A big difference in the Fos expression between mated and $\mathrm{HVF}$-exposed animals was in the MPOA/AH. There were very few Fos-positive nuclei in HVF-exposed animals (Fig. $6 E$ ) compared to a pattern of intense activation in mated animals (Fig. $4 A$ ). Intact HVF-exposed animals also had Fos activity in the caudal pmBNST (Fig. $6 C$ ) similar to that in intact or VNX animals (Fig. 6D) exposed to females. The activation in the rostral pmBNSI and in the MPOA was very low or nonexistent in HVF-exposed animals.

In unilateral VNX animals stimulated with HVF, the accessory olfactory bulb on the intact side had significantly more Fospositive nuclei in both mitral and granule cell layers compared to the VNX side. In the Me, the number of Fos-expressing nuclei on the intact side was almost twice that of the VNX side and the level of activation on the lesion side was comparable to that in unstimulated VNX animals (Fig. $7 A, B$ ). However, in the caudal pmBNST there were equivalent levels of FOS-positive nuclei on both sides. The MPOA and rostral pmBNST (posterior medial bed nucleus of the stria terminalis) had very low levels of expression on both the intact and VNX sides (Fig. $7 B$ ). Bilateral VNX animals had low levels of Fos expression in most brain regions compared even to the lesion side of the unilateral VNX animals. The former animals were also noticeably less active behaviorally than either intact or unilateral VNX animals. They did sniff and lick the HVF but spent less time doing so.

In contrast to the specifically activated regions described above, there was no specific activation of Fos expression, in either mated or HVF-exposed animals, in the central pathways of the main olfactory system: the pyriform cortex, olfactory tubercle, and the anterior cortical or posterolateral amygdala. Both stimulated and unstimulated animals appeared to have the same level of activation. There was also a consistent low level of Fos expression across all groups in other regions of the brain. This was particularly evident in the paraventricular thalamus (Fig. 3 ), but Fos staining was also observed in the main olfactory bulb, lateral septum, and occasionally in parts of the suprachiasmatic nucleus (SCN) in both stimulated and unstimulated animals. The presence or absence of SCN staining may have been due to small differences in the time of day at which the animal room door was opened to move the animals to the clean cage. However, all experiments were started during the first half of the dark phase of the light/dark cycle, and no systematic differences were observed in SCN staining between groups, or in staining of other areas between animals that did or did not show staining in the SCN. The three by two-way ANOVA revealed that both treatment and surgery had significant effects on the number of Fos-positive nuclei in the AOBm (accessory olfactory bulb, mitral cell layer), AOBgr (accessory olfactory bulb, granule cell layer), Me, MPOA, and mBNSTpr (posterior medial bed nucleus of the stria terminalis, rostral). The caudal pmBNST showed a significant effect of treatment but not of VNX surgery. The interaction between treatment and surgery was significant for all other areas except PVN (paraventricular nucleus) thalamus.

\section{Histology}

All VNX animals were examined for residual VN tissue. A few animals showed fragments resembling VN sensory tissue but none appeared to have intact connections passing back toward the AOB. A few nerve bundles ended in neuromata in the VN area as observed in previous experiments (Meredith and O'Connell, 1988). The two VNX animals that mated had no significant residual VN sensory tissue.

\section{Discussion}

Synopsis

We demonstrate here that Fos immunoreactivity in the central vomeronasal pathways (AOB, Me, BNST) and in a vomeronasal target (MPOA) increases dramatically and selectively in mating male hamsters. However, vomeronasal sensory input is not necessary for activation of these regions during mating (except in the case of the AOB), because those few animals that did mate after VNX showed patterns of Fos expression similar, although less intense, to those of mating intact animals. Nevertheless, Fos expression associated with vomeronasal sensory input may be distinguished in the medial amygdala of intact males stimulated with female chemical stimuli (HVF). This expression is 

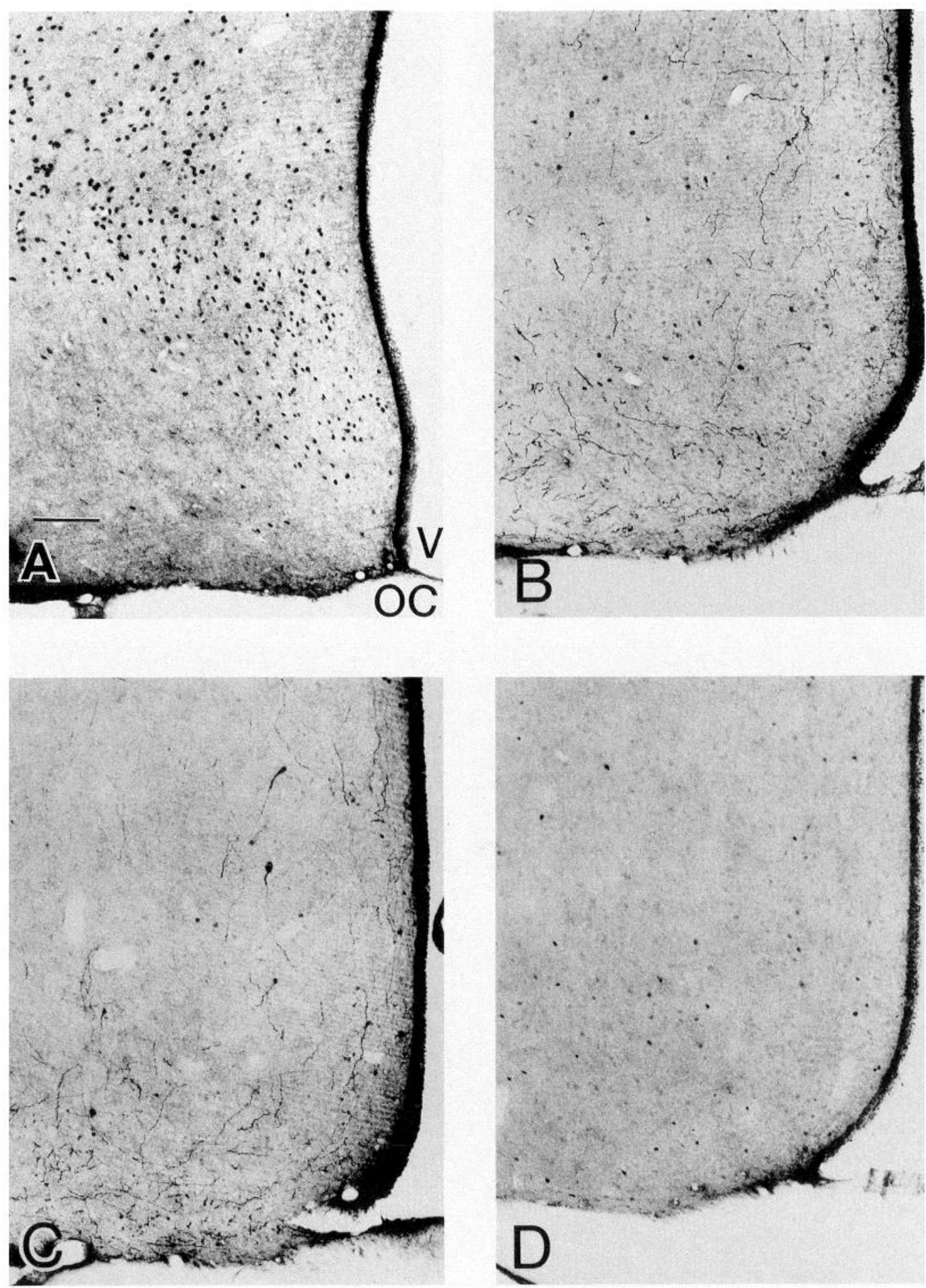

Figure 4. $A-D$, Coronal sections through the medial preoptic area of the four primary groups of animals tested. Note the dense aggregation of Fos-positive nuclei within the MPOA of intact stimulated animals $(A)$. In contrast, VNX stimulated animals that did not mate $(C)$ or unstimulated animals, whether intact $(B)$ or VNX $(D)$ show little activation. The sections shown in $C$ and $D$ were double labeled with LHRH antiserum. $O C$, optic chiasm. See diagram in Figure 5 for location of these sections. Scale bar, $100 \mu \mathrm{m}$.

lacking in VNX animals and, ipsilateral to the lesion, in unilateral VNX animals exposed to HVF. A surprising aspect of these findings is that despite a known requirement for chemosensory input (vomeronasal or olfactory) for male hamster mating behavior (Powers and Winans, 1975; Meredith et al., 1980), there was no equivalent Fos activation in elements of the main olfactory system even of those VNX animals that mated. Fos expression in the caudal part of the pmBNST was associated with the occurrence of chemosensory investigation behavior, regardless of VN status and regardless of whether mating actually occurred, suggesting an involvement of this region in chemosensory investigation. Furthermore, Fos expression in the MPOA/AH and in the rostral pmBNST was limited to those animals that actually mated, again regardless of VN status, sug- 

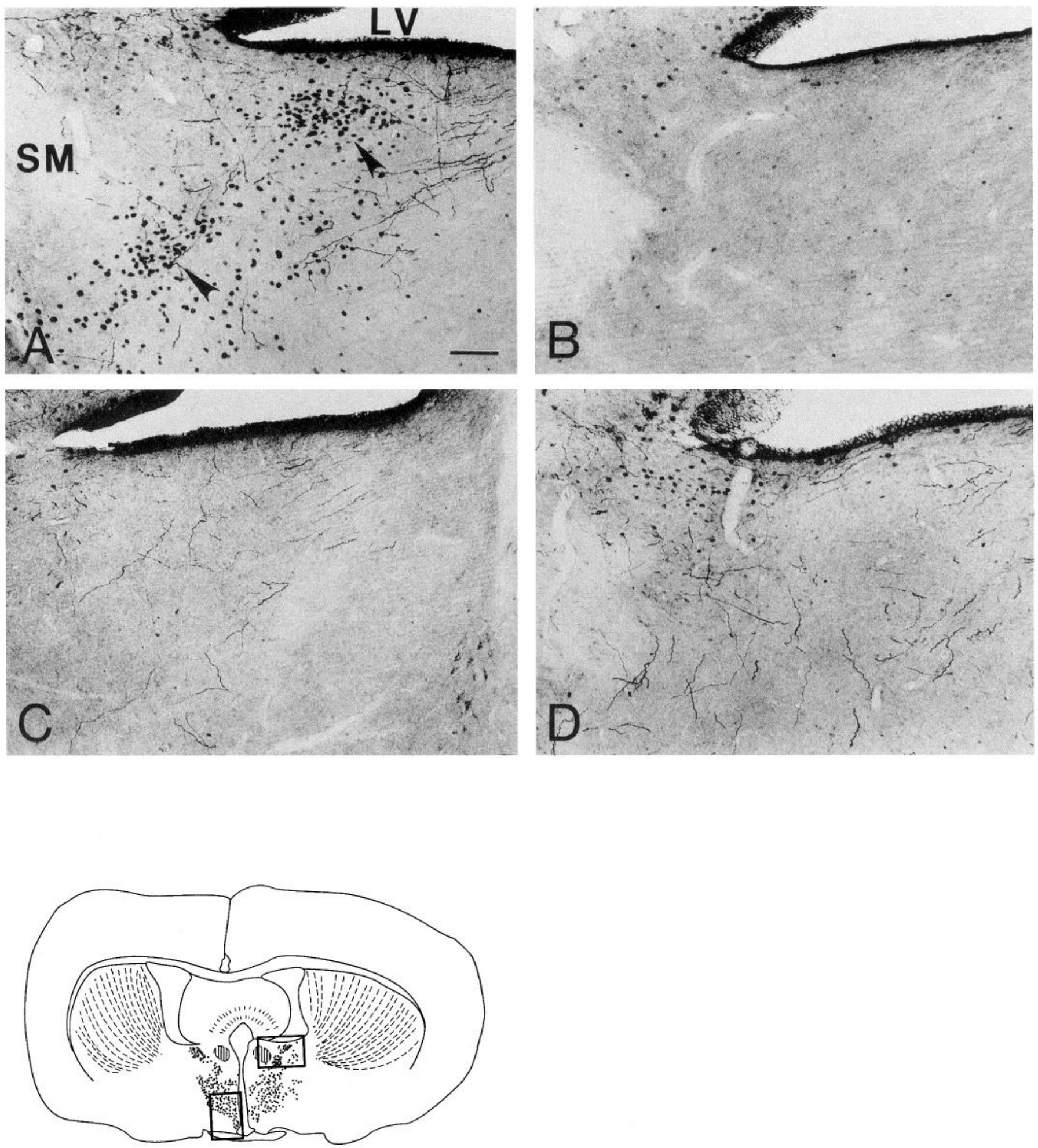

Figure 5. $A-F$, Coronal sections through the posterior medial BNST (rostral) of an intact stimulated animal $(A)$ showing the characteristic pattern of activation (two clusters, arrowheads). VNX stimulated animals $(C)$ or unstimulated animals, whether intact $(B)$ or VNX $(D)$, do not show this activation. The more caudal sections through the pmBNST of both VNX stimulated nonmating animals and intact mated animals had intense Fos expression (see Fig. 6). $A, C$, and $D$ were double labeled for LHRH. $S M$, stria medullaris; $L V$, lateral ventricle. Scale bar, $100 \mu$ m. 

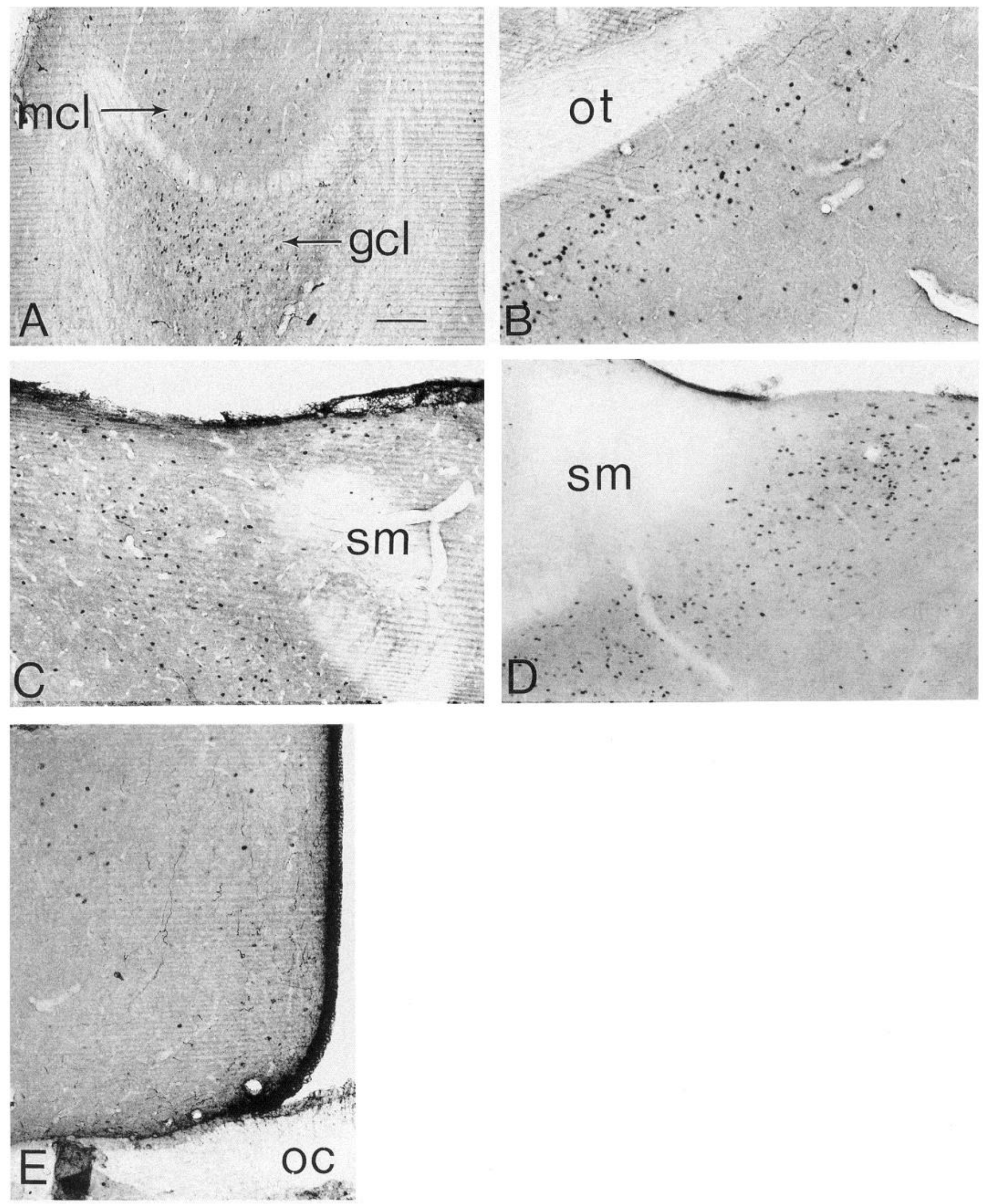

Figure 6. $A-E$, Sections from intact animals stimulated with female hamster vaginal fluid (HVF) at levels shown for animals stimulated with females. $A$, Fos expression in the mitral and granule cell layers in a horizontal section. $B$, Coronal section through posterior medial amygdala. The two clusters characteristic of mated animals are absent. $C$, Coronal section through caudal pmBNST showing a level of Fos expression in this HVFstimulated animal similar to that in intact mated (not shown) and VNX nonmating animals $(D)$. In the MPOA $(E)$ the Fos expression was very low or nonexistent. 

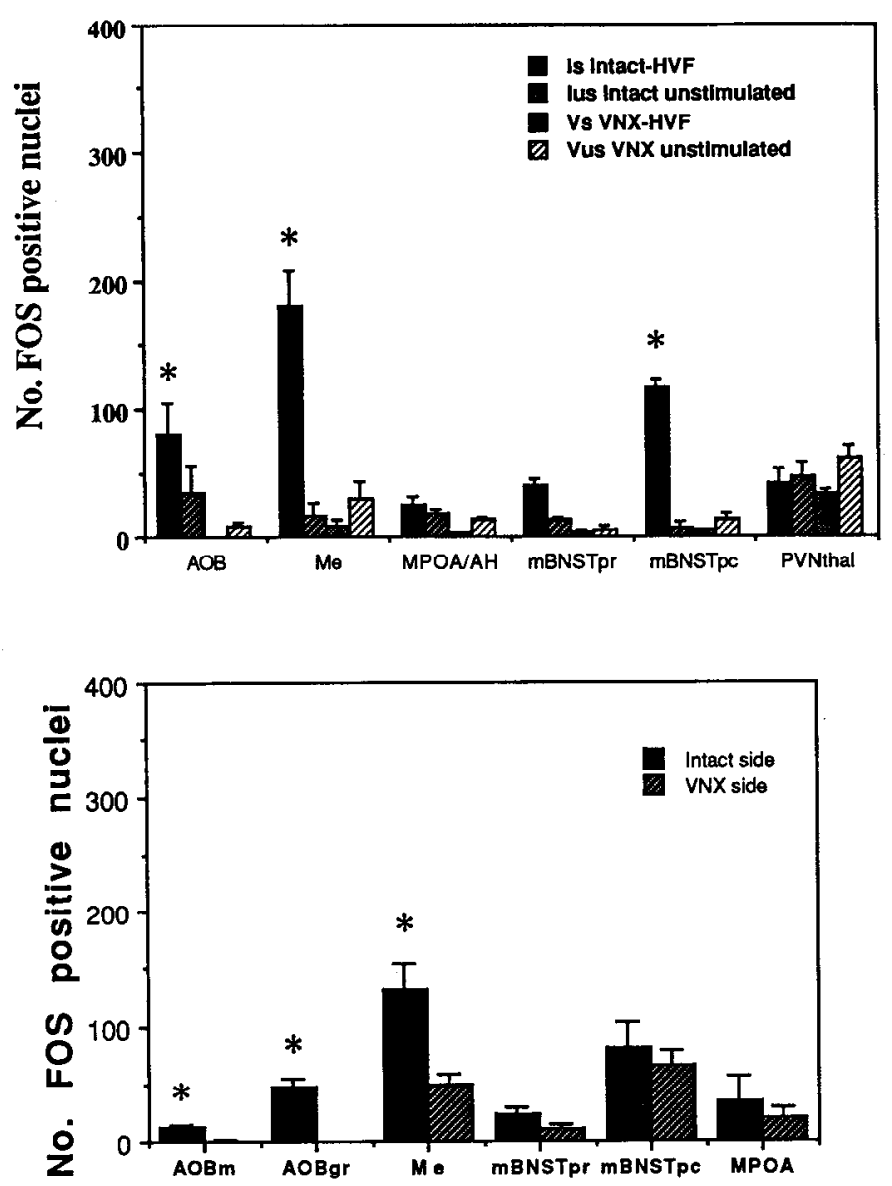

Figure 7. A, Bar graph showing counts of Fos-positive nuclei comparing intact and VNX animals stimulated with female hamster vaginal fluid. Intact HVF-stimulated animals show significantly greater Fos expression $\left({ }^{*}, p<0.02\right.$ ) in the AOB, Me, and the mBNSTpc (posterior medial bed nucleus of the stria terminalis, caudal) compared to unstimulated intact (Ius) and VNX (Vus) controls. There was no increase in the number of activated nuclei in the MPOA. Compared to the counts in mated animals the activation (except in the MPOA and mBNSTpr) is in the same areas but less intense than in mated animals. $B$, Counts of Fos-positive nuclei from unilateral VNX animals stimulated with HVF. The graph shows significant differences between the intact and VNX side in the number of Fos-expressing nuclei within the $\mathrm{AOBm}$, AOBgr, and medial amygdala $\left({ }^{*}, p<0.05\right)$. The intact side had more activation than the VNX side which was barely above the level in VNX unstimulated animals $(A)$. However, within the mBNSTpc the increase in Fos expression was the same on the two sides. There was no increase seen in the MPOA or the mBNSTpr.

gesting that this activation may be concerned with copulatory performance rather than the chemosensory input that initiates copulation.

\section{Selectivity of activation}

In mating animals Fos expression was significantly increased in the AOB and medial amygdala, in both medial and posteromedial cortical nuclei, as well as in the medial BNST, all of which receive output fibers from the $A O B$ and are important components of the central vomeronasal pathway. In contrast, there appeared to be little difference in Fos expression between stimulated and unstimulated animals in central components of the main olfactory system: MOB (main olfactory bulb), pyriform cortex, olfactory tubercle, and anterior cortical and posterior lateral cortical nuclei (PLCN) of the amygdala. Unstimulated intact animals showed very little or no Fos expression in the vomeronasal areas, although these animals did have some Fosexpressing nuclei in other areas, for example, in the paraventricular thalamus (PVNthal) and the main olfactory bulb, but both of these areas appear to be equally activated in all animals (PVNthal, Fig. 3). VNX animals that mated showed activation in the VN pathway (Me and BNST). The selective activation thus appeared to be restricted largely to the central components of the VN pathway but did not appear to depend on vomeronasal sensory input. Intact and unilateral VNX animals stimulated with HVF also showed selective expression in VN pathways despite a lack of mating, so a mating-related activation does not seem to explain the selectivity. Bilateral VNX animals exposed to HVF had very little activation in Me or BNST but this low level may be explained by their low level of investigation of the stimulus. Unilateral VNX animals showed bilateral but asymmetrical expression in the Me but with very low activity on the lesion side, comparable to that in unstimulated VNX animals.

Our results with Fos-expressing neurons in intact stimulated animals are consistent with other studies showing an increase in Fos-expressing neurons in specific neuronal populations in the brain after male mating behavior (Robertson et al., 1991; Baum et al., 1992; Kollack and Newman, 1992; Fiber et al., 1993), but those studies did not include animals with vomeronasal organs removed. Baum et al. (1992) made unilateral olfactory peduncle lesions in rats to eliminate both olfactory and VN input on one side and found a reduction in ipsilateral Fos expression in the Me, except when the males were allowed to mate to ejaculation. This additional mating-related activation on the lesion side must be attributed to either additional sensory input tied to ejaculation or inpul related to the performance itself. Our data on unilateral VNX animals exposed to HVF suggest in addition that a loss of VN input alone significantly reduces chemosensory activation of the ipsilateral Me. The pattern of Fos expression in female rodents after mating is remarkably similar to that in males (Dudley and Moss, 1992), including a significant activation in the MPOA/AH, suggesting an underlying continuity of mechanisms that might not have been expected from the effects of lesions (Pfaff and SchwarzeGiblin, 1988; Sachs and Meisel, 1988). A recent report shows a reduction of Fos expression in LHRH neurons of the medial basal forebrain in VNX, repeatedly mated females (Rajendren et al., 1993), but any VNX-related reduction in Fos expression in other neurons or other brain areas was not reported. We have attempted to identify LHRH neurons (LR1 antibody, courtesy of Dr. R. Benoit) activated during mating behavior in males as part of our investigation of LHRH influence on mating behavior, but have found none. Nor are there any obvious differences in the density of LHRH cells and fibers among the various groups of animals used here. Coolen et al. (1993) also found no Fos activation in LHRH neurons as a result of mating in male rats. It is possible, as proposed by Lambert et al. (1993) for the ferret, that males, unlike females (Rajendren et al., 1993), do not activate LHRH neurons during mating behavior. Given the evidence for an LH response in males exposed to females (Wysocki et al., 1983; Coquelin et al., 1984), it seems more likely that there is activation, but at too low a level to be visible as an increase in Fos expression.

\section{Correlation of Fos expression with behavior}

The pattern of Fos induction after mating behavior in our experiments correlates with a number of lesion studies implicating 
these areas as important in mating behavior. Lesions of the medial amygdala have been shown to eliminate mating behavior in male hamsters (Lehman and Winans, 1982). Lesions in the MPOA have been shown to disrupt copulation, and lesions in the BNST disrupt chemoinvestigative behavior (Powers et al., 1987). In the experiments reported here, most of the VNX stimulated animals did not mate and did not show the activation of VN pathways characteristic of intact animals after mating behavior. This result is consistent with the idea that VN sensory input activates VN pathways relevant to mating behavior. However, in the two VNX animals that did mate, there could be no VN sensory input but the pattern, if not the intensity, of activation in the central VN pathway was essentially the same as in intact mated animals. Counts of Fos-positive nuclei in $\mathrm{AOB}$, Me, BNST, and MPOA were significantly lower in these animals than in intact, mated animals but the relative activation across brain regions was similar (other than $\mathrm{AOB}$ ) in intact mating and VNX mating animals. Finally, activation in the MPOA and rostral pmBNST was very low in all HVF-exposed animals despite the fact that the Me activation in intact and unilateral VNX animals exposed to HVF reached levels comparable to that in VNX animals that mated (when given access to females). The presence or absence of MPOA and rostral pmBNST activity is correlated here as clscwhere with occurrence of mating. Thus, activity in MPOA and rostral pmBNST appears related to mating performance. In the more caudal part of the pmBNST (caudal encapsulated BNST) there was a consistent activation in all animals that were stimulated by females regardless of whether they mated or not and regardless of their VN status. Thus, VNX animals that did not mate but did investigate females and intact or unilateral VNX animals that investigated chemical stimuli (HVF) showed Fos expression in caudal pmBNST similar to that in intact or VNX animals that mated. The bilateral VNX animals exposed to HVF did not show a significant increase in Fos expression in the caudal pmBNST, but this is not very surprising since these animals did not perform much chemoinvestigatory behavior compared to $\mathrm{VNX}$ animals exposed to receptive females or unilateral VNX animals exposed to HVF. In unilateral VNX animals stimulated with HVF, significant differences in Fos expression between the intact and VNX sides were seen in the AOB and Me. The caudal pmBNST, on the other hand, had equivalent levels of activation on both sides. Unstimulated animals showed very little activity in any part of the BNST. Fos expression in the caudal pmBNST appears not to be dependent on vomeronasal input but does depend on chemosensory investigation, suggesting that this region either directs or responds to chemosensory investigation.

The consistent Fos expression in MPOA and in rostral pmBNST in mating animals but not in HVF-exposed animals or in nonmating VNX animals has already been noted above. A relationship between levels of mating performance and Fos expression in the MPOA has also been noted before (Baum and Everitt, 1992), where successive restriction of performance produced a successive decrease in Fos expression within the MPOA. These findings suggest that MPOA and rostral pmBNST activation may relate more to the copulatory performance or to the sensory input dependent on copulation than to the chemosensory input that initiates it.

\section{$V N$ sensory input}

The lack of Fos expression in most VNX animals, in any of the regions characteristically activated in mating intact animals, does not provide enough data to distinguish between : os activation by $\mathrm{VN}$ sensory input and activation as a conseq ence of mating behavior itself. The two VNX animals that $\mathrm{d} 1$ mate, both of which were confirmed histologically to have no significant residual VN tissue, as well as the animals stimulated with HVF are important for analyzing chemosensory influence on Fos expression. The level of Fos expression in mitral and granule cell layers of the AOB in VNX mating animals was no greater than that in the VNX stimulated animals that failed to mate, consistent with the histological absence of VNOs. However, the level of Fos expression in other areas, especially the medial amygdala, was at an intermediate level, being significantly lower than in the intact stimulated group but significantly higher than in the VNX stimulated nonmating group. The additional level of Fos activation in intact mating animals compared to VNX mating animals may be attributed to $\mathrm{VN}$ sensory input. Unilateral VNX animals exposed to HVF investigated the stimulus intensely but had significantly less activation in the AOB and Me ipsilateral to the lesion. This difference is presumably due to a loss of VN input. The higher levels of Fos expression in mating animals compared with HVF-exposed intact or unilateral VNX animals may be attributed to mating-related input, either from sensory or from centrally generated activation. Thus, there appears to be a VN input that is supplemented in mating animals by additional activation, perhaps generated centrally or perhaps by other sensory input. Baum and Everitt's (1992) observation of additional input to Me on the side of a unilateral olfactory peduncle lesion in animals allowed to ejaculate is consistent with this interpretation. Some of the increment in Fos expression associated with mating could arise from males licking and grooming their own genital area between intromissions, a consistent part of the mating pattern in rodents. In intact animals this could also be a source of additional VN input. We know that inexperienced VNX animals as a group have severely impaired mating behavior. As here, very few will mate on the first exposure to females (Meredith, 1986), so VN input is important for the behavior. Our evidence here suggests VN input is responsible for Fos expression in the $A O B$, and that part of the Fos expression in the $\mathrm{Me}$ in response to females or their chemosignals also derives from VN input.

\section{Conclusion}

In conclusion, central vomeronasal pathways do seem to be activated selectively during mating behavior and exposure to HVF; and input through the VN system appears to contribute to that activity in VNO intact animals. In contrast, the main olfactory system did not show mating- or stimulation-spccific activation, and both stimulated and unstimulated animals showed equivalent amounts of Fos expression in the main olfactory bulbs. Fos expression in MPOA and rostral pmBNST is limited to those animals that engaged in mating behavior with a female. Chemosensory input was not sufficient for this activation. On the other hand, activation in caudal pmBNST appeared in intact and VNX animals that performed chemosensory investigative behavior regardless of whether they mated. VN sensory input is known to be important for mating behavior in male hamsters but VN sensory input appears not to be necessary for initiation of some of the characteristic patterns of Fos expression in VN pathways seen in intact mated animals. A more detailed analysis of the vomeronasal and other contributions to activation within central target nuclei and their projections appears warranted from the data presented here. 


\section{References}

Barber PC, Raisman G (1974) An autoradiographic investigation of the projection of the vomeronasal organ to the accessory olfactory bulb of the mouse. Brain Res 81:21-30.

Baum MJ, Everitt BJ (1992) Increased expression of c-fos in the medial preoptic area after mating in malc rats: role of afferent inputs from the medial amygdala and midbrain central tegmental field. Neuroscience 3:627-646.

Baum MJ, Wersinger SR (1993) Equivalent levels of mating-induced neural c-fos immunoreactivity in castrated male rats given androgen, estrogen or no steroid replacement. Biol Reprod 48:1341-1347.

Beltramino C, Taleisnik S (1983) . Release of LH in the female rat by olfactory stimuli (Effect of the removal of the vomeronasal organs or lesioning of the accessory olfactory bulbs). Neuroendocrinology 36 : 53-58.

Beltz BS, Burd GD (1989) Immunocytochemical techniques-principles and practice. Blackwell.

Clancy AN, Macrides F, Singer AG, Agosta WC (1984) Male hamster copulatory responses to a high molecular weight fraction of vaginal discharge-cffects of vomeronasal organ removal. Physiol Behav 33: 653-660.

Coolen JMM, Peters JPW, Veening JG (1993) Increased expression of c-fos following sexual behavior in male rats in relation to the LHRH system. Psychoneuroendocrinology, in press.

Coquelin A, Clancy AN, Macrides F, Nobel EP, Gorski RA (1984) Pheromonally induced release of luteinizing hormone in male mice. Involvement of the vomeronasal system. J Neurosci 4:2230-2236.

Davis BJ, Macrides F, Youngs WM, Schneider SP, Rosene DL (1978) Efferents and centrifugal afferents of the main and accessory olfactory bulbs in the hamster. Brain Res Bull 3:59-72.

Dudley CA, Rajendran G, Moss RL (1992) Induction of Fos immunoreactivity in central olfactory structures of the female rat following exposure to conspecific males. Mol Cell Neurosci 3:360-369.

Fernandcz GD, Mcredith M (1991) Early vomeronasal lesions cause severe deficits in male hamster mating behavior, relieved in part by intracerebral LHRH peptides. Chem Senses 16:519-520.

Fernandez GD, Meredith M (1992) Vomeronasal pathways are selectively activated during mating behavior in male hamsters. Soc Neurosci Abstr 18:1202.

Fiber JM, Adames P, Swann JM (1993) Pheromones induce c-fos in limbic areas regulating male hamster mating behavior. Neuroreport $4: 871-874$.

Hines M, Allen LS, Gorski RA (1992) Sex differences in subregions of the medial nucleus of the amygdala and the bed nucleus of the stria terminalis of the rat. Brain Res 579:321-326.

Johnston RE (1977) Sex pheromones in golden hamsters. In: Chemical signals in vertebrates (Muller Schwarze D, Mozell MM, eds), pp 225249. New York: Plenum.

Kevetter G, Winans SS (1981a) Connections of the corticomedial amygdala in the golden hamster. I. Efferents of the vomeronasal amygdala. J Comp Neurol 197:81-98.

Kevetter G, Winans SS (1981b) Connections of the corticomedial amygdala in the golden hamster. II. Efferents of the olfactory amygdala. J Comp Neurol 197:99-101.

Kollack SS, Newman SW (1992) Mating behavior induces selective expression of Fos protein within the chemosensory pathways of the male Syrian hamster brain. Neurosci Lett 143:223-228.

Krettek JE, Price JL (1978) A description of the amygdaloid complex in the rat and cat with observations on the intra-amygdaloid axonal connections. J Comp Neurol 178:255-279.

Lambert GM, Rubin BS, Baum MJ (1993) Sex difference in the effect of mating in luteinizing hormone-releasing hormone neurons of the ferret forebrain. Endocrinology 131:1473-1480.

Lehman MN, Winans SS (1982) Vomeronasal and olfactory pathways to the amygdala controlling male hamster sexual behavior: autoradiographic and behavioral analyses. Brain Res 240:27-41.

I ehman MN, Winans SS (1983) Evidence for a ventral non-strial pathway from the amygdala to the bed nucleus of the stria terminalis in the male golden hamster. Brain Res 268:139-143.

Licht GA, Mcrcdith M (1987) Convergence of main and accessory oltactory pathways onto single neurons in the hamster amygdala. Exp Brain Res 69:7-18.

Lloyd-Thomas A, Keverne EB (1982) Role of the brain and accessory olfactory system in the block to pregnancy in mice. Neuroscience 7:907-911.

Lomas DE, Keverne EB (1982) Role of the vomeronasal organ and prolactin in the acceleration of puberty in female mice. $J$ Reprod Fertil 66:101-107.

Meredith M (1980) The vomeronasal organ and accessory olfactory system in the hamster. In: Chemical signals in vertebrates and aquatic animals (Muller Schwarze D, Silverstein RM, eds), pp 199-252. New York: Academic.

Meredith M (1983) Sensory physiology of pheromone communication. In: Pheromones and reproduction in mammals (Vandenbergh JG, ed), pp 199-252. New York: Academic.

Meredith M (1986) Vomeronasal organ removal before sexual experience impairs male hamster mating behavior. Physiol Behav 36:737743.

Meredith M, O'Connell R (1988) HRP uptake by olfactory and vomeronasal receptor neurons: use as an indicator of incomplete lesions and relevance for non-volatile chemoreception. Chem Senses 13:487515 .

Morgan JI, Cohen DR, Hempstead JL, Curran T (1987) Mapping patterns of c-fos expression in the central nervous system after seizure. Science 237:192-197.

Pfaff DW, Schwarze-Giblin S (1988) Cellular mechanisms of female reproductive behaviors. In: The physiology of reproduction, Vol 2 (Knobil E, Neill JD, eds), pp 1487-1568. New York: Raven.

Powers JB, Winans SS (1975) Vomeronasal organ: critical role in mediating sexual behavior of the male hamster. Science 187:961963.

Powers JB, Newman SW, Bergondy ML (1987) MPOA and BNST lesions in male Syrian hamsters: differential effects on copulatory and chemoinvestigatory behaviors. Behav Brain Res 23:181-195.

Kajendren G, Dudley CA, Moss RL (1993) Influence of male rats on the luteinizing hormone releasing hormone neuronal system in female rats: role of the vomeronasal organ. Neuroendocrinology 57:898-906.

Reynolds J, Keverne EB (1979) The accessory olfactory system and its role in pheromonally mediated suppression of oestrus in grouped mice. J Reprod Fertil 57:31-35.

Robertson GS, Pfaus JG, Atkinson LJ, Matsumura H, Phillips AG, Fibiger HC (1991) Sexual behavior increases c-fos expression in the forebrain of the male rat. Brain Res 564:352-357.

Sachs BD, Meisel RL (1988) The physiology of male sexual behavior. In: The physiology of reproduction, Vol 2 (Knobil E, Neill JD, eds), pp 1393-1485. New York: Raven.

Sagar SM, Sharp FR, Curran T (1988) Expression of c-fos protein in the brain: metabolic mapping at the cellular level. Science 240:13281331 .

Saito TR, Moltz H (1986) Sexual behaviour in the female rat following removal of the vomeronasal organ. Physiol Behav 38:81-87.

Sanchez-Criado JE (1982) Involvement of the vomeronasal system in the reproductive physiology of the rat. In: Olfaction and endocrine regulation (Breiphol W, ed), pp 209-221. London: IRL.

Scalia F, Winans SS (1975) The differential projections of the olfactory bulb and accessory bulb in mammals. J Comp Neurol 161:31-56.

Scalia F, Winans SS (1976) New perspectives on the morphology of the olfactory system-olfactory and vomeronasal pathways in mammals. In: Mammalian olfaction, reproductive processes and behavior (Doty RL, ed), pp 8-28. New York: Academic.

Siegel S (1956) Nonparametric statistics for the behavioral sciences. Tokyo: McGraw-Hill Kogakusha.

Wysocki CJ, Meredith M (1987) The vomeronasal system. In: Neurobiology of taste and smell (Finger TE, Silver WL, eds), pp 125-150. New York: Wiley.

Wysocki CJ, Nyby J, Whitney G, Beauchamp GK, Katz Y (1982) The vomeronasal organ: primary role in mouse chemosensory gender recognition. Physiol Behav 29:315-327.

Wysocki CJ, Katz Y, Bernhard R (1983) Male vomeronasal organ mediates female induced testosterone surges in mice. Biol Reprod 28 : 917-922. 